\title{
The Performance of Electron Counting Direct Detection in Electron Energy Loss Spectroscopy
}

\author{
Shaobo Cheng ${ }^{1}$, Paolo Longo ${ }^{2}$, Ray D. Twesten ${ }^{2}$ and Gianluigi A. Botton ${ }^{1, *}$ \\ 1. Canadian Centre for Electron Microscopy and Department of Materials Science and Engineering, \\ McMaster University, Hamilton, Canada. \\ 2. Gatan Inc., Pleasanton, CA, USA. \\ * Corresponding author: gbotton@mcmaster.ca
}

In recent decades, transmission electron microscopy (TEM), and electron energy loss spectroscopy (EELS) in particular, have seen many advances [1,2]. Meanwhile, direct electron detection has been widely used for imaging beam sensitive materials, including the biomaterials, polymers, etc $[3,4]$. One would instinctively wonder which improvements would be apparent when direct electron detection (DeD) is used to acquire EELS spectra. Early reports have shown the benefits for EELS in a conventional microscope [5]. Taking advantage of the DeD hardware (Gatan K2 IS), we have systematically studied the performance of the K2 IS for the acquisition of EELS spectra at different accelerating voltages on an FEI Titan 80-300 Cubed TEM equipped with a monochromator and two aberration correctors. We have carried out the experiments with the monochromator excited, in order to understand the behaviors of the K2 IS when optimal energy resolution is needed. Using single crystalline $\mathrm{SrTiO}_{3}$ as model system, the point spread functions, modulation transfer functions, signal-to-noise ratio, detective quantum efficiency of the K2 IS detector have been evaluated.

The EELS acquisition system consists of two detectors installed in a Gatan Quantum Energy Filter: the conventional CCD (US 1000) and K2 IS Summit which can be selected independently from each other. Since two detectors are installed in the same spectrometer, it is easy to control the same experimental conditions (such as beam current, same observation area on specimen). Since the K2 IS provides fast acquisition speed (400 frames/second) and small dynamic range, it is suitable for acquiring core loss spectra. Our initial results show that the K2 IS has overall better performance than the CCD at $200 \mathrm{kV}$ while at 80 $\mathrm{kV}$, the $\mathrm{K} 2$ detector still provides much higher signal to noise but exhibits slightly broader broader tails of the zero-loss peak (Figure 1) resulting in sharper and less noisy core loss spectra (Figure 1). This information needs to be accounted for when selecting the optimal detector and operating conditions.

Furthermore, four types of noise sources have been quantitively analyzed, including Fano noise, shot noise, gain noise, read-out noise. The signal-to-noise ratio has been calculated for both CCD and K2 under different acquisition conditions. Moreover, we have characterized the performance of the K2 vs CCD in atomicresolved imaging for high energy features, such as the $\mathrm{Sr} \mathrm{L}_{2,3}$ edge, including the energy resolution expected when a large acquisition energy range is needed (Figure 2). The results show the remarkable improvements for weak signals when atomic resolution (hence small currents) is required. Systematic measurements related to: dwell time, energy dispersion, signal to noise, energy range, and detector quantum efficiency at different voltages have been carried out and will be presented.

In summary, using $\mathrm{SrTiO}_{3}$ as a model system, we have the evaluated the performance of $\mathrm{K} 2$ and $\mathrm{CCD}$ working at different accelerating voltages over a range of acquisition parameters. While providing high acquisition speed, the DeD provides significant advantages for materials science applications such as detecting trace elements or analyzing beam sensitive samples [6].

References:

[1] S. Cheng, et al., Science Advances 4 (2018) eaar4298. 
[2] X. Li, et al., Advanced Materials Interfaces 6 (2018), p. 1701246.

[3] X. Li, et al., Nature Methods 10 (2013), p. 584.

[4] Y. Zhu, et al., Nature Materials 16 (2017), p. 532.

[5] J. L. Hart, et al., Scientific Reports 7 (2017), p. 8243.

[6] G. A. B and S. C. are grateful to NSERC for a Discovery Grant partially supporting this work. G. A. B is grateful to the Canada Foundation for Innovation, under the JELF program, for supporting the acquisition of the K2 IS detector. Electron microscopy was carried out at the Canadian Centre for Electron Microscopy (CCEM), a national facility supported by NSERC, the Canada Foundation for Innovation, under the MSI program, and McMaster University.
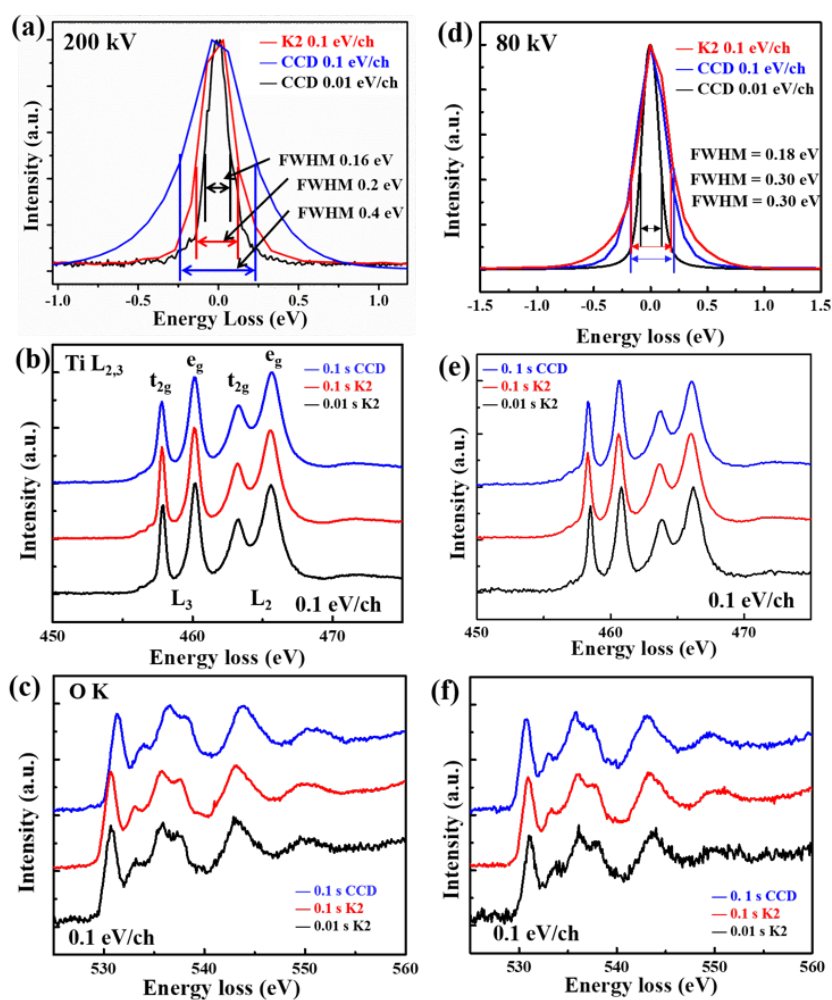

Figure 1. The EELS spectra acquired on single crystalline $\mathrm{SrTiO}_{3}$ sample. (a) The zero loss peaks (ZLP) acquired by $\mathrm{K} 2$ and $\mathrm{CCD}$. The energy resolution for the system is estimated to be $0.16 \mathrm{eV}$. The full width at half maximum (FWHM) values for $\mathrm{K} 2$ and CCD with $0.1 \mathrm{eV} / \mathrm{ch}$ dispersion are $0.2 \mathrm{eV}$ and $0.4 \mathrm{eV}$, respectively. (b) $\mathrm{Ti} \mathrm{L}_{2,3}$ edges acquired by $\mathrm{K} 2$ and CCD with $0.1 \mathrm{eV} / \mathrm{ch}$ dispersion. $\mathrm{T}_{2 \mathrm{~g}}$ peaks acquired by K2 are narrower than that acquired by CCD. (c) The corresponding EELS spectra for O K edges. Spectra in (a)-(c) were acquired at $200 \mathrm{kV}$. (d) ZLPs acquired by different detectors with different dispersions under 80 $\mathrm{kV}$. The ZLP acquired by K2 has broader tails. (e)(f) Ti L 2,3 edges and $\mathrm{O} \mathrm{K}$ edges acquired with $0.1 \mathrm{eV} / \mathrm{ch}$ dispersion under $80 \mathrm{kV}$.
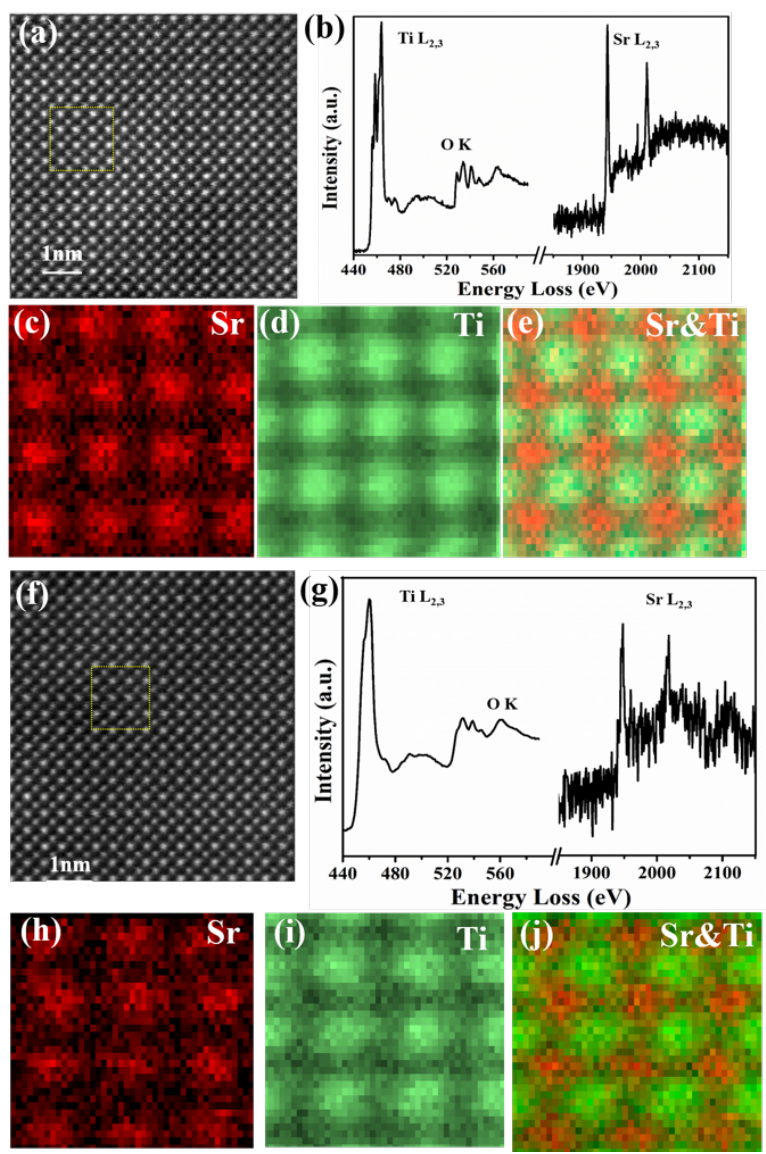

Figure 2. The EELS mapping results for $\mathrm{SrTiO}_{3}$ on the [100] zone axis at $200 \mathrm{kV}$ with $0.1 \mathrm{~s} /$ pixel acquisition time. (a) HAADF images for $\mathrm{SrTiO}_{3}$. The area for EELS mapping is marked by yellow rectangle. (b) $\mathrm{Ti}_{2,3}$ edges, $\mathrm{O} \mathrm{K}$ edge and $\mathrm{Sr}_{2,3}$ edges extracted from K2 EELS mapping result. The $\mathrm{e}_{\mathrm{g}} / \mathrm{t}_{\mathrm{gg}}$ splitting can be seen. (c)-(e) EELS mapping results for $\mathrm{Sr}$ and $\mathrm{Ti}$ and the combination signal of $\mathrm{Sr}$ and $\mathrm{Ti}$. (f) HAADF image acquired before EELS mapping. The yellow rectangle area shows the mapping area for $\mathrm{CCD}$. (g) Ti $\mathrm{L}_{2,3}$ edges, $\mathrm{O} \mathrm{K}$ edge and $\mathrm{Sr}_{2,3}$ edges extracted from the CCD EELS mapping results. More noise can be observed. The $e_{g}$ and $t_{2 g}$ splitting cannot be separated. (h)-(j) Atomic scale mapping results for $\mathrm{Sr}$ and $\mathrm{Ti}$ and the combination of $\mathrm{Sr}$ (red) and $\mathrm{Ti}$ (green). The mapping result for $\mathrm{Sr}_{2,3}$ edges is not as clear as that shown in (c) because of the reduced signal-to-noise ratio. 